DOI: 10.18027 / 2224-5057-2017-7-3-66-72

Цитирование: Коломиец Л. А., Чернышова А. Л., Чуруксаева О. Н., Виллерт А. Б., Молчанов С. В. Оптимизация современных подходов в лечении гинекологического рака основных локализаций // Злокачественные опухоли. - 2017. - Т. 7. - №3, спецвыпуск 1. - С. 66-72.

\title{
Оптимизация современных подходов в лечении гинекологического рака основных локализаций
}

\author{
Л. А. Коломиец, А. Л. Чернышова, О. Н. Чуруксаева, А.Б. Виллерт, С. В. Молчанов
}

\begin{abstract}
Резюме: Проблема злокачественных опухолей женской репродуктивной системы продолжает оставаться актуальной в связи с отсутствием значимой динамики со стороны показателей эффективности лечения и выживаемости, а также четкой тенденцией омоложения данного контингента больных. В настоящей статье представлены основные задачи, решение которых оказывает влияние не только на эффективность проводимого противоопухолевого лечения, но и решает вопросы оптимизации объемов лечения, качества жизни и репродукции. Концепция сторожевых лимфатических узлов обретает особую ценность у категории больных, претендующих на органосохраняющее лечение при раке шейки матки. Применение нового отечественного РФП 99mTc-Al²03 демонстрирует его эффективное накопление по сравнению с импортным аналогом, а его практическое применение позволяет облегчить интраоперационное выявление сторожевых лимфатических узлов. С целью прогнозирования течения местно-распространенного рака шейки матки возможно использование ряда тканевых маркеров: высокий уровень Кi-67 до неоадювантной химиотерапии и гиперэкспрессия COX-2 и белка p16ink4a после проведения неоадъювантной химиотерапии коррелируют с неблагоприятным прогнозом. У больных с диссеминированным раком яичников определение индекса PIV и выполнение лапароскопии на первом этапе позволит объективизировать показания для проведения оптимальной и полной циторедукции. Не менее важным моментом является возможность прогнозирования эффективности химиотерапии у больных раком яичников. Детекция мутаций генов BRCA 1 и 2 лежит в основе персонализации лечения при раке яичников. В группе, представленной 178 больными раком яичников, выявлено 24 образца, гетерозиготных по мутациям BRCA 1,2 (13,6\%). Чаще всего присутствовали мутации BRCA15382insC ( $n=10,42 \%)$ и BRCA14153delA ( $n=8,33 \%)$.
\end{abstract}

Ключевые слова: рак шейки матки, рак яичников, сторожевые лимфатические узлы, факторы прогноза, Кі-67, С0Х-2, p16ink4a, лапароскопия, индекс PIV, мутации генов BRCA1,2

\section{Информация об авторах}

Лариса А. Коломиец, НИИ онкологии Томского НИМЦ, e-mail: kolomietsla@oncology.tomsk.ru

Алена Л. Чернышова, НИИ онкологии Томского НИМЦ, e-mail: alacher@list.ru

Ольга Н. Чуруксаева, НИИ онкологии Томского НИМЦ, e-mail: churuksaevaon@mail.ru

Алиса Б. Виллерт, НИИ онкологии Томского НИМЦ, e-mail: avillert@yandex.ru

Сергей В. Молчанов, НИИ онкологии Томского НИМЦ, e-mail: sergemolchanov@yandex.ru

В настоящее время удельный вес гинекологического рака основных локализаций (рак шейки матки, эндометрия и яичников) составляет 45,5\% в структуре злокачественных новообразований органов репродуктивной системы и отличается увеличением доли женщин репродуктивного возраста, что определяет новые тенденции в развитии онкогинекологии - повышение качества жизни этой категории больных и сохранение у них репродуктивного потенциала [1]. В связи с этим вопросы органосохраняющего лечения рака шейки матки в течение многих десятилетий продолжают оставаться в центре внимания ведущих отечественных и зарубежных онкологов. Предпосылками к разработке методов лечения начального инвазивного рака шейки матки с сохранением репродуктивной функции явились особенности канцерогенеза, а именно редкость поражения яичников и редкость распространения на тело матки [2]. Согласно рекомендациям NCCN Guidelines Version 1.2017 Cervical Cancer при стадиях IA1-IB рекомендовано применение органосохраняющего лечения в объеме радикальной трахелэктомии с тазовой 
лимфодиссекцией с определением сторожевых лимфатических узлов. Поскольку абсолютное число больных, являющихся потенциальными кандидатами для органосохраняющего лечения, достаточно велико, это обуславливает разработку новых подходов к лечению РШМ с сохранением фертильности [2, 3]. Потенциально радикальная абдоминальная трахелэктомия разрабатывалась как адекватная альтернатива расширенным операциям у пациенток с микроинвазивным и инвазивным РШМ при размерах опухоли до $2 \mathrm{~cm}$. Основное отличие радикальной трахелэктомии (РТ) от радикальной гистерэктомии при сопоставимых размерах новообразования заключается в том, что при РТ сохраняются тело матки, яичники и маточные трубы [4]. Только в этом случае могут быть соблюдены принципы онкологической радикальности наряду с сохранением фертильности.

Поскольку метастатическое поражение регионарных лимфатических узлов является определяющим фактором прогноза у операбельных больных раком шейки матки (РШМ) и имеет ведущее значение в распространении процесса, важное место при выполнении радикальной трахелэктомии уделяется подвздошно-тазовой лимфодиссекции и методам оценки состояния регионарных лимфатических узлов. Риск лимфогенной диссеминации опухоли при РШМ IА стадии в регионарные лимфатические узлы составляет 2,8-4,8\%, при IB - 13,6-15,9\%, тактика лечения может быть основана на результатах экспресс-морфологического, или послеоперационного исследования удаленных лимфатических узлов [5]. Многие авторы справедливо подчеркивают, что удаление лимфатических узлов не приносит пользу большинству пациенток с начальными стадиями рака шейки матки. Лимфаденэктомия увеличивает продолжительность операции и, возможно, кровопотерю, повышает риск повреждения магистральных сосудов, мочеточников, бедренно-полового нерва, лимфедемы и образования лимфатических кист. Суммарная частота других тяжелых осложнений не превышает $6 \%$.

На сегодняшний день точность методов диагностики функционального статуса лимфатических узлов является недостаточной, в связи с чем концепция биопсии сигнальных лимфоузлов приобретает все большее признание и введена для многих локализаций опухолей в стандарты хирургического лечения Европейской организацией по изучению и лечению рака (EORTC).

В 2014 году в Российской Федерации для визуализации СЛУ в Томском НИИ онкологии и Томском политехническом университете в рамках проекта № 16. №8.12.1011 «Доклинические исследования нового лимфотропного радиофармацевтического препарата на основе меченого технецием-99m гамма-оксида алюминия» (ФЦП «Развитие фармацевтической и медицин-ской промышленности Российской Федерации на период до 2020 года и дальнейшую перспективу») был разработан оригинальный РФП на основе меченого технецием-99m гамма-оксида алюминия (99mTc-Al²O3) [6].

Экспериментальное изучение фармакокинетики радиофармпрепарата на основе меченого технецием-99m гамма-оксида алюминия показало, что исследуемый РФП может успешно использоваться для лимфосцинтиграфии и визуализации сторожевых узлов с 15 минуты и до 24 часов после его подкожного введения. Учитывая уровень радиационной защиты современных гамма-зондов, который превосходит $0,1 \%$, указанные соотношения (1,5-6,5\%) вполне приемлемы для эффективного интраоперационного выявления сторожевых лимфатических узлов с использованием исследуемого РФП [6].

Экспериментальное изучение нового отечественного РФП 99mTc-Al²O3 показало, что разработанный наноколлоид в несколько раз активнее накапливается в лимфатических узлах по сравнению с импортным аналогом и его практическое применение позволит облегчить интраоперационное выявление СЛУ. Нами проведено исследование, в которое вошло 30 больных раком шейки матки IA-IB стадии, которым проводилось органосохраняющее лечение в объеме радикальной абдоминальной трахелэктомии с определением СЛУ с применением РФП 99mТс$\mathrm{Al}^{2} \mathrm{O} 3$. Получены первые данные клинического применения разработанного радиофармпрепарата, которые свидетельствуют о высоких показателях чувствительности 94\% и специфичности 100\%, что позволит выявлять сторожевые лимфатические узлы при раке шейки матки с достаточно высокой эффективностью (Патент № 2535614 от 15.10.2014). Концепция СЛУ особенно ценна в тех случаях, когда статус лимфатических узлов является наиболее важным фактором целесообразности проведения органосохраняющего лечения, но требует дальнейшего изучения.

Еще одной особенностью РШМ в настоящее время является увеличение удельного веса местно-распространенного рака шейки матки (МРРШМ), причем от $30 \%$ до 45\% больных местно-распространенным РШМ погибают в течение первых пяти лет от прогрессирования основного заболевания, поэтому проблема поиска надежных прогностических критериев остается актуальной $[7,8]$. Прогностические факторы при онкологическом заболевании часто являются более значимыми в плане исхода заболевания, чем терапевтический эффект. Рецепторы гормонов, биохимические маркеры, экспрессия онкогенов и антигенов, связанная с пролиферацией, а также другие молекулярные маркеры повсеместно признаны прогностически значимыми. В нашем исследовании были изучены возможности маркера пролиферативной активности Ki-67, белка p16ink4a, являющегося ингибитором циклинзависимых киназ, и циклооксигеназы COX-2 в определении прогноза течения местно-распространенного РШМ. Материалом исследования служила ткань опухоли шейки матки 25 больных МРРШМ. Средний возраст пациенток составил 38,04ะ1,6 лет. Оценивалась первоначальная экспрессия перечисленных иммуногистохимических марке- 
ров в опухоли и после проведения 2-х курсов платино-содержащей неоадъювантной химиотерапии (НАХТ).

Было выявлено, что индекс пролиферации в среднем у больных МРРШМ составил $60 \%$. Высокий и очень высокий уровень экспрессии (более 50\%) наблюдался у $64 \%$ больных МРРШМ, слабый уровень экспрессии Кі-67 отмечался в $16 \%$. Полученные результаты согласуются с данными авторов, утверждающих, что уровень пролиферативной активности плоскоклеточного рака шейки матки предопределяет отдаленные результаты лечения, а также является предсказательным фактором для определения чувствительности к химио- и лучевой терапии $[9,10]$. Кроме того, имеются данные о том, что пролиферативная активность эпителиальных клеток при предраковых поражениях шейки матки зависит от наличия ВПЧ ВКР [11]. В то же время есть исследования, не обнаруживающие такой зависимости и указывающие, что пролиферативная активность при РШМ может варьировать в широких пределах, отличаясь у разных больных более чем в 10 раз [12]. Ряд авторов предлагает определять экспрессию PCNA, Ki-67 и p16ink4a, служащих суррогатными маркерами экспрессии вирусных онкогенов, с целью предсказывания активности вирусных генов E6/E7 [13, 14, 15].

Анализ экспрессии циклооксигеназы СОХ-2 показал, что экспрессия СОХ-2 выявлялась у больных МРРШМ в 76\% случаев. Высокий уровень экспрессии был диагностирован у $20 \%$ больных, низкий уровень экспрессии наблюдался в $56 \%$ случаев. Средний уровень экспрессии СОХ-2 составил $26,3 \%$.

Полученные данные об экспрессии СОХ-2 не противоречат результатам других авторов [16]. Ряд исследователей сообщает о вовлеченности СОХ-2 в цервикальный канцерогенез и свидетельствуют об ассоциации этого маркера с распространенными стадиями заболевания, плохим прогнозом и рецидивами [10, 17]. В других работах значимость СОХ-2 как фактора прогноза отрицается [18]. Одни авторы обнаруживают корреляционную связь между экспрессией СОХ-2 и ВПЧ, утверждая, что онкобелки Е6 и Е7 увеличивают транскрипцию COX-2 через EGFR-сигнальный путь, другие ученые указывают на отсутствие связи экспрессии СОХ-2 с наличием ВПЧ-инфекции $[12,19]$.

Еще одним из прогностических факторов, ассоциированным с худшим течением РШМ, рассматривают повышенную экспрессию белка p16ink4a, продуцируемого геном INK4a, который принадлежит к группе ингибиторов циклинзависимых киназ. Нами было выявлено, что достоверно чаще белок p16ink4a экспрессировался в клетках больных МРРШМ (80\%). Уровень экспрессии в среднем у этих пациенток составил 47\%. Проанализирована взаимосвязь ВПЧ-инфекции с белком p16ink4a. Полученные результаты свидетельствуют, что в большинстве случаев экспрессия белка p16ink4a сочетается с наличием ВПЧ-инфекции онкогенных типов. У больных МРРШМ такое сочетание наблюдается в $68 \%$ случаев. Возможно, что гиперэкспрессия этого белка у ВПЧ-инфицированных больных объясняется взаимодействием белкового продукта онкогена Е7 ВПЧ $\mathrm{c} \mathrm{Rb}$, что приводит к инактивации последнего. Доказано, что в свою очередь по механизму обратной связи усиливается транскрипция гена, кодирующего белок p16ink4a [20].

В настоящее время исследования показывают, что появление клеток, экспрессирующих белок p16ink4a, отмечается уже на ранних стадиях опухолевого процесса. Так, при раке in situ наблюдается четкий сдвиг в сторону фокального и диффузного окрашивания, которое является преобладающим в инвазивных карциномах. В то же время отсутствие экспрессии р16INK4а в ткани плоскоклеточной карциномы, возможно, указывает на иной механизм развития последней, не ассоциированный с ВПЧ [21]. Было выявлено, что присутствие генома HPV высокого риска не является необходимым условием для осуществления экспрессии белка p16ink4a в опухолевых клетках. Этот белок отмечался и в тканях больных раком молочной железы, легкого и мочевого пузыря. В то же время причины повышенной экспрессии до конца не выявлены.

С целью изучения прогностического значения параметров Ki-67, COX-2 и p16INK4a была проанализирована динамика этих показателей у больных МРРШМ до начала лечения и после проведения 2-х курсов ПХТ. Анализ иммуногистохимических маркеров, определяемых до начала противоопухолевого лечения и после 2-х курсов НАХТ у больных МРРШМ, выявил достоверное снижение удельного веса опухолей шейки матки, экспрессирующих Кi-67, циклооксигеназу COX-2 и ингибитор циклинзависимых киназ белок p16ink4a после завершения курсов химиотерапии. Показано, что если до начала лечения показатель пролиферативной активности Кі-67 выявлялся у всех больных МРРШМ, то после курсов НАХТ 24\% пациенток не экспрессировали этот антиген. Среднее его значение уменьшилось с 60\% до 42\% (p<0,05), то есть менее половины опухолевых клеток находилось в митотическом периоде клеточного цикла. Уровень высокой и очень высокой экспрессии Кі-67 снизился в 2 раза по сравнению с показателями до лечения: с $64 \%$ до $32 \%$.

Отчетливая экспрессия циклооксигеназы COX-2, оказывающей влияние на подавление апоптоза, активацию неоангиогенеза, способствующей адгезии опухолевых клеток, выявлялась в 76\% случаев у первичных больных МРРШМ. После проведения НАХТ отмечается достоверное снижение удельного веса опухолей, экспрессирующих СОХ-2, до 40\%, причем в 36\% наблюдается слабая экспрессия - до 25\%.

Анализ экспрессии белка ингибитора циклин-зависимых киназ p16ink4a после проведения НАХТ показал его достоверное снижение. До лечения p16ink4a экспрессировался у 80\% пациенток МРРШМ, после лечения - у 52\% больных. Также отмечается снижение уровня экспрессии: если до лечения экспрессия более 50\% отмечалась у 40\% больных МРРШМ, то после лекарственной терапии высокая и очень высокая экспрессия выявлялась в 2 раза реже. 
В работе была проанализирована связь Кі-67, СОХ-2, p16ink4a с отдаленными результатами лечения у больных МРРШМ. Было выявлено, что у больных МРРШМ с уровнем экспрессии Кі-67 менее 50\% показатели 5-летней общей выживаемости статистически значимо превышают аналогичные показатели больных МРРШМ с экспрессией Ki-67 > 50\% (Gehan'sWilcoxon p=0,01056, Log-RankTest $\mathrm{p}=0,00899$, Cox-MantelTest $\mathrm{p}=0,01011)$. В группе больных, у которых экспрессия Кі-67 была ниже 50\%, 5-летняя общая выживаемость составила 100\%, среди пациенток с высоким уровнем пролиферативной активности $($ Кi-67 > 50\%) - 67\%. Безрецидивная 5-летняя выживаемость у пациенток МРРШМ, независимо от уровня экспрессии Кі-67, статистически значимо не различалась. Значимой взаимосвязи исходных уровней СОХ-2 и p16ink4a у больных МРРШМ с отдаленным результатами лечения выявлено не было. Однако установлено, что экспрессия этих показателей после проведения НАХТ сопряжена с отдаленными результатами лечения. Так, 5-летняя выживаемость больных МРРШМ при уровне экспрессии СОХ-2 < 50\% составила $84 \%$, а при экспрессии более $50 \%$ - 66\%.

Анализ зависимости безрецидивной выживаемости от уровня экспрессии белка p16ink4a после НАХТ показал, что безрецидивная выживаемость больных МРРШМ, у которых экспрессии данного белка не отмечалось, статистически значимо превышала аналогичный показатель выживаемости тех пациенток, у которых отмечалась высокая и очень высокая экспрессия этого белка. Показатель 3-хлетней безрецидивной выживаемости у p16ink4a-отрицательных больных составил 50\%, у пациенток с низкой экспрессией этот показатель составил 40\%, а у больных с высокой экспрессией - 25\%. Пятилетняя безрецидивная выживаемость у больных МРРШМ с отсутствием экспрессии и низким ее уровнем не отличалась и достигала 20\%, а больных с высокой экспрессией белка p16ink4a в этом сроке наблюдения не было. Аналогичные результаты получены другими авторами [10]. Отсутствие достоверных различий в ряде случаев, вероятно, может быть связано с небольшим количеством пациенток, что предполагает продолжить исследования в данном направлении.

Таким образом, высокий уровень пролиферативной активности Кі-67 у первичных больных, гиперэкспрессия COX-2 и белка p16ink4а после проведения неоадъювантной химиотерапии коррелируют с неблагоприятным прогнозом. Высокие уровни экспрессии Кі-67 и СОХ-2 статистически значимо влияют на 5 -летнюю общую выживаемость, а уровень экспрессии белка p16ink4a сопряжен с показателями безрецидивной выживаемости. Результаты проведенного исследования указывают на важное значение изученных параметров и подтверждают их предиктивную роль в прогнозе МРРШМ.

Рак яичников на протяжении последних десятилетий продолжает оставаться наиболее высокомортальной онкогинекологической патологией. Распределение больных
РЯ по стадиям достоверно отличается от их распределения при раке шейки матки и раке эндометрия, характеризуясь вдвое меньшей долей встречаемости I-II стадий среди больных с впервые в жизни установленным заболеванием и преобладанием III-IV стадии заболевания

B 2002 г. в исследовании Bristow R.Е. было показано, что наиболее важным критерием, оказывающим влияние на прогноз данной нозологии, является наличие и размер остаточной опухоли в результате циторедуктивного вмешательства [22]. В связи с этим актуальным является вопрос использования НАХТ у ряда пациенток с распространенным раком яичников.

В 2013 г. проведен мета-анализ, включивший 1220 случаев рака яичников III-IV ст. [23]. Было показано, что статистически значимой разницы медианы общей и безрецидивной выживаемости у пациенток с первичной циторедуктивной операцией и пациенток, проходивших НАХТ, нет. В резюме отмечено, что тактика ведения пациентки (первичная циторедуктивная операция или НАХТ) выбирается индивидуально в каждом случае. В тоже время на сегодняшний день не существует объективных критериев дооперационной оценки возможности выполнения оптимальной или полной циторедукции. В связи с этим ведется поиск шкал интраоперационной оценки возможности выполнения полной циторедукции, наиболее востребованной из которых на данный момент является Total Predictive Index Value (PIV), которая была предложена в 2006 г. Было показано, что при PIV $\geq 8$ вероятность резекции R0 практически равна 0 , соответственно, рекомендовано проведение НАХТ [24]. Кроме того нет четких рекомендаций о применении лапароскопии в диагностике и лечении этой категории больных. Нами было проведено исследование по определению роли лапароскопии в оценке выраженности степени перитонеального канцероматоза с подсчетом PIV при хирургическом стадировании диссеминированного рака яичников. В группе больных с первичной циторедуктивной операцией среднее значение индекса PIV составило 5,4, в группе с первичной НАХТ - 10,3, которое снизилось до 4,8 перед промежуточной операцией. Анализ характера циторедуктивной операции по группам в результате предложенного алгоритма выявил, что в 1 группе удельный вес субоптимальных составил 28,6, 3\%, оптимальных - 42,8\%, полных - 28,6\%, во второй группе -29,4\%, 35,3\%, 35,3\% соответственно.

Таким образом, выполнение лапароскопии с определением индекса PIV на первом этапе позволяет объективизировать показания для проведения оптимальной и полной циторедукции у больных диссеминированным раком яичников.

Не менее важным моментом является возможность прогнозирования эффективности химиотерапии у больных раком яичников. Эффективность применения «золотого стандарта» - паклитаксела в сочетании с препаратами платины - в качестве терапии 1-й линии у больных 
с распространенным РЯ составляет 70-80\%, включая 40\% полных регрессий $[25,26]$. В то же время у большинства женщин впоследствии возникает рецидив заболевания. Доля опухолей, первично резистентных к стандартным схемам терапии первой линии с включением препаратов платины, составляет 15-20\% случаев. Все это указывает на целесообразность усовершенствования старых и разработки новых стандартов лечения РЯ в будущем. До сих пор нерешенными остаются задачи, связанные с учетом особенностей, специфичных для каждой пациентки, к которым следует в первую очередь отнести молекулярногенетические факторы (на уровне организма и опухоли). Выделить заранее группу больных с резистентным к стандартной терапии течением на основании лишь клинических данных практически невозможно. В литературе последних десятилетий имеются многочисленные данные об уровне отдельных показателей, свидетельствующих о нарушении механизмов пролиферации, апоптоза и ангиогенеза в опухолевых клетках при РЯ.

Модель овариального канцерогенеза, предложенная Kurman R.J., не является заменой гистопатологической классификации овариальных карцином, однако привлекает внимание к молекулярно-генетическим событиям, которые играют роль в прогрессии опухоли. В настоящее время ни один из молекулярно-генетических параметров, представленных в концепции канцерогенеза, не используется на практике и не определяет тактику и выбор химиотерапевтического воздействия. При этом резистентность к применяемым при раке яичников препаратам платины может быть обусловлена многими факторами, включая снижение активного транспорта препаратов, повышенную активность процессов их элиминации, быструю детоксикацию путем конъюгации с глутатионом, повышенную устойчивость к повреждениям ДНК, уменьшение способности обнаружения повреждений ДНК, ускоренное выведение ДНК-платиновых соединений, усиленную репарацию ДНК и патологические сигналы к апоптозу, часто связанные с потерей подавляющего рост опухоли белка P53, но также и не зависящие от Р53. Патогенез развития рака яичников, ассоциированного с мутациями генов BRCA 1 и 2, характеризуется развитием событий по 2 пути молекулярно-генетической классификации овариальных карцином. Мутации в гене BRCA1 накладывают особенности на его клиническое течение и чувствительность к химиотерапии. Современные возможности лечения наследственного РЯ, ассоциированного с мутациями генов BRCA 1 и 2, ограничены небольшим спектром химиопрепаратов, действие которых основывается на взаимосвязи с обусловленными мутациями, дефектами репарации ДНК. Скомпрометированная данными мутациями способность опухолей восстанавливать двунитевые разрывы ДНК объясняет более высокую чувствительность к ДНК-повреждающим агентам, в том числе платиновым производным. Также имеется лучший ответ на доксорубицин и гемцитабин, чем у больных с отсутствием мутаций, а также митомицин С. В целом считается, что пациентки с мутациями BRCA1/2 обладают более благоприятным прогнозом [27].

Помимо этого, опухоли с BRCA1- или BRCA2-мутациями, являются чувствительными к блокаде нормального восстановления однонитевых разрывов ДНК с участием PARP через PARP-ингибирование. При наличии мутаций в генах BRCA1/2 и PARP-ингибировании возникает феномен «синтетической летальности», когда полностью теряется способность восстановления ДНК в опухолевых клетках BRCA-ассоциированного рака. Ингибирование PARP может быть особенно эффективно как у BRCA-мутированных больных, так и у больных с нарушениями процессов репарации ДНК, обусловленных другими причинами, что составляет примерно половину случаев $\mathrm{HG}$-опухолей яичника. Таким образом, эффективность применения данной группы препаратов гораздо выше частоты встречаемости герминальных мутаций гена BRCA. Известно, что на долю синдрома рака молочной железы и яичников («hereditary breast-ovarian cancer») приходится до 15$20 \%$ случаев РЯ. До 90\% врожденных генетических нарушений представлено мутациями в генах BRCA1 и BRCA2. Риск заболеть РМЖ у носительниц мутантного гена достигает 85-90\%, а РЯ - 50\% [28]. Спектр инактивирующих мутаций в генах BRCA характеризуется исключительным разнообразием, поэтому диагностика наследственного синдрома требует полного секвенирования упомянутых генов. Доказано, что в некоторых регионах мира, в том числе и в РФ, значительно выражен так называемый эффект предшественника (founder эффект). Подавляющую часть BRCA мутаций составляют варианты BRCA15382 insC и BRCA14153 delA. В России, Польше, Венгрии наиболее частой является мутация 5382 insC в гене BRCA1. Наследственный фактор присутствует у $41 \%$ евреек-ашкенази, страдающих РЯ, у которых обнаруживается три типа генеративных мутации: BRCA1185AG, BRCA15382insC, BRCA26174delT с общей частотой 2,5\% (по сравнению c 0,1-0,3\% в общей популяции женщин [29].

В данное исследование были включены 178 больных РЯ (23-85 лет). Средний возраст больных с мутацией составил 48,74ะ7,9 лет. Удельный вес больных с диссеминированным РЯ был высоким: частота встречаемости III C стадии соответствовала 76,3\%, IV стадии - 24\%. Исследование BRCA-статуса проводилось с использованием аллель-специфической ПЦР в режиме реального времени. В 178 образцах ДНК, выделенных из крови больных РЯ, выявлено 24 образца, гетерозиготных по мутациям BRCA (13,6\%). Чаще всего встречалась мутация BRCA15382insC $(n=10,42 \%)$ и BRCA14153delA $(n=8,33 \%)$, значительно реже (в общей сложности 21\%) - BRCA1 185delAG ( $\mathrm{n}=1)$, BRCA1 T300G $(n=1)$, BRCA12080delA $(n=1)$, BRCA2 $(n=2)$.

Таким образом, на данный момент нет сомнений, что РЯ является абсолютным показанием для молекулярно-генетического исследования. Перспективным направлением, 
связанным с решением вопросов персонализации лечения РЯ, является оптимизация молекулярно-генетических прогностических маркеров дефектов репарации ДНК в опухоли. Поддерживающая терапия PARP-ингибитора- ми у больных рецидивным РЯ с мутациями генов BRCA является эффективным способом увеличения показателей эффективности лечения при отсутствии значимого влияния на качество жизни больных.

\section{Литература • References}

1. Состояние онкологической помощи населению России в 2016 году. Под. ред. А. Д. Каприна, В. В. Старинского, Г.В.Петровой. - М.: МНИОИ им. П. А. Герцена - фрилиал ФГБУ «НМИРЦ» Минздрава России, 2017. - $236 \mathrm{c}$.

2. Wuntakal R, Papadopoulos AJ, Montalto SA, Perovic M, Coutts M, Devaja O. Int J. Location of Sentinel Lymph Node in Cervical Carcinoma and Factors Associated With Unilateral Detection. Gynecol Cancer. 2015 Nov;25 (9):1663-8.

3. Ungar, L. Surgical treatment of lymph node metastases in stage IB cervical cancer. The laterally extended parametrectomy (LEP) procedure: experience with a 5 year follow-up/L. Ungar, L. Palfalvi, L. Tarnai et al. // Gynecol. Oncol. - 2011. - Vol. 123, №2. P. 337-41.

4. Zhang, D. A new method of surgical margin assuring for abdominal radical trachelectomy in frozen section/D. Zhang, H. Ge, J. Li et al.// J. of Cancer. - 2015. - Vol. 51, №6. - P. 734-41.

5. Zada, A. Meta-analysis of sentinel lymph node biopsy in breast cancer using the magnetic technique/A. Zada, M. C. L. Peek, M. Ahmed et al. // Br J Surg. - 2016. - Vol. 103, №11. - P. 1409-19.

6. Варламова Н. В., Скуридин В.С., Нестеров Е. А., Чернов В. И., Тицкая А.А. Исследование острой токсичности нового радиосрармацевтического препарата «Наноколлоид, 99mTc-Al203» для диагностики в онкологии. // Экспериментальная и клиническая фрармакология. 2015. Т. 78. №6. С. 26-29.

7. Карапетян В. Л., Степанова Е. В., Барышников А. Ю., Никогосян С. О., Кузнецов В. В. Молекулярно-биологические факторы прогноза рака яичников начальных стадий // Вестник РОНЦ им. Н. Н. Блохина РАМН, т. 22, №1, 2011 - С. 37-40.

8. Samir Raghad, Asplund Anna, Tot Tibor, Pekar Gyula, Hellberg Dan Tissue tumor marker expression in smokers, including serum cotinine concentrations, in women with cervical intraepithelial neoplasia or normal squamous cervical epithelium // Am J Obstet Gynecol 2010; 202: 579. e1-7. doi: 10.1016/j. ajog. 2009.11.034.

9. Винокуров В.Л., Пожарисский К. М., Жаринов Г. М., Гаспарян Н. А., Самсонова Е. А., Кузнецова М. Е., Болдарян Н. А. Иммуногистохимические маркеры в качестве прогностических критериев в онкогинекологии // Вопросы онкологии. - 2008. - №4. - С. 463-470.

10. Кузнецова М. Е. Иммуногистохимическая оценка пролиферативной активности и репаративных способностей плоскоклеточного рака шейки матки как показателей эфффективности лучевой терапии: диссертация... кандидата медицинских наук Санкт-Петербург, 2009. - 84 c.

11. Cambruzzi E., Zettler C. G., Alexandre C. 0. Expression of Ki-67 and squamous intraepithelial lesions are related with HPV in endocervical adenocarcinoma // P Cambruzzi E. athology Oncology Res. 2005. V. 11. P. 114-120.

12. Carriho C., Gouveia P., Cantel M. [Et al.] Characterization of human papillomavirus infection, p53 and ki-67 expression in cervix cancer of Mozambican women // Pathol. Res. Pract. - 2003. - Vol. 199. - P. 303-311.

13. Naucler P., Ryd W., Tornberg S. et al. Efficacy of HPV DNA testing with cytology triage and/or repeat HPV DNA testing in primary cervical cancer screening. J Natl Cancer Inst 2009; 101 (2):88-99. doi: 10.1093/jnci/djn444. Epub 2009 Jan 13.

14. Ozgul N, CilA P, Bozdayi G, Usubutun A, Bulbul D, Rota S, Kose MF, Biri A, Haberal A Staining characteristics of p16INK4a: is there a correlation with lesion grade or high-risk human papillomavirus positivity?/J Obstet Gynaecol Res. 2008 0ct;34 (5):865-71.

15. Mitchell A, Newton JM, Brite K, Einspahr J, Ellis M, Davis J, et al. Cyclooxygenase 2 expression in cervical intraepithelial neoplasia and vulvar cancer. J Low Genit Tract Dis 2007; 11 (2):80-5.

16. Kiviat N. B., Hawes S. E., Feng Q. Screening for cervical cancer in the era of the HPV vaccine - the urgent need for both new screening guidelines and new biomarkers. J Natl Cancer Inst 2008; 100:290-1. doi: 10.1093/jnci/djn038. Epub 2008 Feb 26.

17. Dursun P, Yuce K, Usubutun A, Ayhan A. Cyclooxygenase-2 expression in cervical intraepithelial neoplasia III and squamous cell cervical carcinoma, and its correlation with clinicopathologic variables. Int J Gynecol Cancer 2007; 17 (1):164-73.

18. Gadducci A., Guerrieri ME, Greco C. Tissue biomarkers as prognostic variables of cervical cancer Crit Rev Oncol Hematol 2013 May;86 (2):104-29. doi: 10.1016/j. critrevonc. 2012.09.003. Epub 2012 Sep 30.

19. Kuo KT, Chang HC, Hsiao CH et al. Increased ki-67 proliferative index and absence of p16INK4a in CIN-HPV related pathogenic pathways different from cervical squamous intraepithelial lesion//British J Ophthalmol - 2006 - Vol. 90 - p. 894-899. 
20. Sales KJ, Katz AA, Howard B, Soeters RP, Millar RP, Jabbour H. Cyclooxygenase-1 is up-regulated in cervical carcinomas: autocrine/paracrine regulation of Cyclooxygenase-2, prostoglandine receptors, angiogenic factors by Cyclooxygenase-1// Cancer Res. - 2002; 62: 424.

21. Hammes Luciano S., Tekmal Rajeshwar Rao, Naud Paulo, Edelweiss Maria Isabel, KirmaNameer, Valente Philip T., Syrjanen Kari J., Cunha-Filho Joao Sabino Up-regulation of VEGF, c-fms and COX-2 expression correlates with severity of cervical cancer precursor (CIN) lesions and invasive disease// Gynecologic Oncology 110 (2008) 445-451. doi: 10.1016/j. ygyno. 2008.04.038. Epub 2008 Jun 20.

22. Bristow R. E., Tomacruz R. S., Armstrong D. K., Trimble E. L., Montz F. J. Survival effect of maximal cytoreductive surgery for advanced ovarian carcinoma during the platinum era: a meta-analysis. Journal of Clinical Oncology. 2002. Vol. 20. P. 1248-59.

23. Dai-yuan M., Bang-xian T., Xian-fu L. Ye-qin Z., Hong-Wei C. A meta-analysis: neoadjuvant chemotherapy versus primary surgery in ovarian carcinoma FIGO stagelll and IV. World Journal of Surgical Oncology. 2013. https://doi.org/10.1186/1477-7819-11-267. doi: 10.1186/1477-7819-11-267.

24. Fagotti A., Ferrandina G., Fanfani F. et al. A laparoscopy-based score to predict surgical outcome in patients with advanced ovarian carcinoma: a pilot study. Annals of Surgical Oncology. 2006. Vol. 13. P. 1156-61.

25. Ozols R. F., Bundy B. N., Greer B. E., Fowler J. M., Clarke-Pearson D., Burger R. A., Mannel R. S., DeGeest K., Hartenbach E. M., Baergen R. Phase III Trial of Carboplatin and Paclitaxel Compared With Cisplatin and Paclitaxel in Patients With Optimally Resected Stage III Ovarian Cancer: A Gynecologic Oncology Group Study // Journal of Clinical Oncology 200321:17, 3194-3200.

26. Vasey P. A. Resistance to chemotherapy in advanced ovarian cancer: mechanisms and current strategies // Br J Cancer. $2003 \mathrm{Dec}$; 89 (Suppl 3): S23 - S28.

27. Bolton et al. Association between BRCA1 and BRCA2 mutations and survival in women with invasive epithelial ovarian cancer // JAMA, 2012;307 (4): 382-39. doi: 10.1001/jama. 2012.20.

28. Easton D. F., Ford D., Bishop D. T. Breast and ovarian cancer incidence in BRCA1-mutation carriers. Breast Cancer Linkage Consortium // Am J Hum Genet. 1995 Jan;56 (1):265-71.

29. Robles-diaz L., Goldfrank D. J, Kauff N. D, Robson M., Offit K. Hereditary ovarian cancer in Ashkenazi Jews // Familial Cancer, 2004, Volume 3, Number 3-4, P. 259. 